\title{
Robust observer-based fault estimation and accommodation of discrete-time piecewise linear systems
}

\author{
Tabatabaeipour, Mojtaba; Bak, Thomas
}

Published in:

Journal of the Franklin Institute

Link to article, DOI:

10.1016/j.jfranklin.2013.08.021

Publication date:

2013

Link back to DTU Orbit

Citation (APA):

Tabatabaeipour, M., \& Bak, T. (2013). Robust observer-based fault estimation and accommodation of discretetime piecewise linear systems. Journal of the Franklin Institute, 351(1), 277-295.

https://doi.org/10.1016/j.jfranklin.2013.08.021

\section{General rights}

Copyright and moral rights for the publications made accessible in the public portal are retained by the authors and/or other copyright owners and it is a condition of accessing publications that users recognise and abide by the legal requirements associated with these rights.

- Users may download and print one copy of any publication from the public portal for the purpose of private study or research.

- You may not further distribute the material or use it for any profit-making activity or commercial gain

- You may freely distribute the URL identifying the publication in the public portal

If you believe that this document breaches copyright please contact us providing details, and we will remove access to the work immediately and investigate your claim 


\title{
Robust observer-based fault estimation and accommodation of discrete-time piecewise linear systems
}

\author{
Seyed Mojtaba Tabatabaeipour ${ }^{\mathrm{a}, *}$, Thomas Bak ${ }^{\mathrm{b}}$ \\ ${ }^{\mathrm{a}}$ Automation and Control, Department of Electrical Engineering, Technical University of Denmark, \\ DK-2800 Kgs. Lyngby, Denmark \\ ${ }^{\mathrm{b}}$ Department of Electronic Systems, Aalborg University, DK-9220 Denmark
}

Received 22 February 2013; received in revised form 21 June 2013; accepted 23 August 2013

\begin{abstract}
In this paper a new integrated observer-based fault estimation and accommodation strategy for discretetime piecewise linear (PWL) systems subject to actuator faults is proposed. A robust estimator is designed to simultaneously estimate the state of the system and the actuator fault. Then, the estimate of fault is used to compensate for the effect of the fault. By using the estimate of fault and the states, a fault tolerant controller using a PWL state feedback is designed. The observer-based fault-tolerant controller is obtained by the interconnection of the estimator and the state feedback controller. We show that separate design of the state feedback and the estimator results in the stability of the overall closed-loop system. In addition, the input-tostate stability (ISS) gain for the closed-loop system is obtained and a procedure for minimizing it is given. All of the design conditions are formulated in terms of linear matrix inequalities (LMI) which can be solved efficiently. Also, performance of the estimator and the state feedback controller are minimized by solving convex optimization problems. The efficiency of the method is demonstrated by means of a numerical example.
\end{abstract}

(c) 2013 The Franklin Institute. Published by Elsevier Ltd. All rights reserved.

\section{Introduction}

There is an increasing demand on safety, reliability and performance of modern industrial systems. A fault in the system might deteriorate the performance of the system or lead to the loss

\footnotetext{
*Corresponding author. Tel.: +45 253570.

E-mail address: setaba@elektro.dtu.dk (S.M. Tabatabaeipour).
} 
of the system functionality or stability. In some instances, it might result in hazardous events. Therefore, it is very important to design control systems that can tolerate occurrence of some faults during the operation while guaranteeing stability and functionality of the system and maintaining an acceptable performance. Such controllers are called fault-tolerant. The area of fault-tolerant control (FTC) has attracted a lot of attentions in the past two decades, see review papers $[2,18,11]$ and books $[9,3]$.

Broadly speaking, FTC systems are divided into two categories: passive (PFTC) and active (AFTC). In PFTC, the FTC system does not react to the occurrence of a fault in the sense that the structure and parameters of the controller are pre-designed and fixed such that it can tolerate a set of faults without any change in the controller. This means that the fault tolerant controller provides a common solution to the problem of control design for the normal system as well as the faulty systems. Therefore, the PFTC solution is usually a conservative solution. Moreover, when some severe faults are taken into account, a common solution may not always exist and if it exists, it usually yields a low performance. On the other hand in AFTC, the controller reacts to the occurrence of faults and changes the parameters and/or the structure of the controller. A fault detection and estimation module is used to detect and estimate the fault when it occurs. Then, based on the information about the occurred fault, a supervisory controller changes the control law or the structure of the controller, in the case of severe faults, such that the faulty system with the new controller is stable and provides an acceptable performance. AFTC can usually provide a better performance because it changes or modifies the controller based on the characterizations of the occurred fault.

In the past three decades many methods have been developed for AFTC and PFTC of discreteevent or continuous systems. For a review of these methods, the interested reader is referred to [3]. But many of the industrial systems include both discrete and continuous behaviors. These class of systems are called hybrid systems. Hybrid systems are systems that include both continuous and discrete behaviors and a non-trivial interaction between continuous evolution and discrete transitions. Generally speaking, a hybrid system consists of several modes of behavior. In each mode the system has a continuous dynamic. Transition between these modes might be for example state-dependent, time- dependent, input dependent or based on occurrence of some external events or a combination of these conditions. Hybrid systems appear in many practical engineering applications such as mechanical systems, chemical processes, embedded systems, manufacturing and traffic control.

FTC of hybrid systems has attracted some research in recent years. For example see the book by Yang and Jiang [29]. But the research in this area is still at its infancy and there is a need for more research in this area. PWL systems are an attractive class of hybrid systems. This is because PWL framework proposes an efficient way to describe the dynamic of systems exhibiting switching between a number of linear systems where switching is state-dependent [13,7]. In many nonlinear systems this switching is because of PWL components such as dead-zone, saturation, hysteresis, etc. These nonlinearities appear in many industrial applications and can be efficiently modeled by a PWL system. For example, in [26] it is shown that many practical systems such as mechanical motion systems with friction can be efficiently modeled as PWL systems. Moreover, PWL systems can approximate nonlinear systems effectively [21]. Furthermore, in [7] it is shown that discrete-time piecewise affine systems are equivalent to other classes of hybrid systems such as mixed logical dynamical systems and linear complementary systems under mild well-posedness assumption. Also, system identification methods such as $[24,6,19]$ can be used to identify a PWL model of a nonlinear system. PWL systems have been recently used for modeling and control of systems in different application 
domains. For example in [27] a PWL model of a pitch controlled wind turbine is obtained using clustering based techniques. In [22,8] PWL systems are used for modeling and control of prostate cancer. In [23] a PWL model of a live-stock building is used for control and reconfigurability analysis.

Closely related to PWL systems are switched systems. An important problem in stability of switched systems is determining restrictions on minimum time between switches (dwell-time), see [4,5] and references therein. Ref. [28] considers the problem of fault-tolerant control for nonlinear switched systems with periodic switching signal. Authors assume that the switching sequence is fixed and there is a series of prescribed dwell-times between switches. A PWL system can be viewed as a switched linear system where switching is based on the partitioning of the state space. In PWL systems switching occurs if the state of the system enters a new partition meaning that the switching times and period between them cannot be assumed fixed or given a priori since they depend on the dynamic of the system in each mode.

There are very few methods available in the literature for fault detection and fault-tolerant control of PWL systems. Motivated by this fact, in this paper, we address the problem of fault estimation and fault tolerant control for PWL systems. Fault tolerant control of PWL systems using model predictive control by means of mixed integer programming is investigated in [17]. A mixed logical dynamical modeling framework is used for modeling of hybrid systems and the problem is solved using mixed integer optimization. However, these approaches suffer from high computational complexity. In [25], a guaranteed cost PFTC method for piecewise affine (PWA) systems in discrete-time using piecewise Lyapunov functions is proposed. The controller can tolerate partial loss of actuator gains while guaranteeing a specific level of performance. Sufficient conditions in terms of LMIs for the existence of the controller are given. An important step in design of an AFTC is to check if the system is reconfigurable with respect to a given fault. Reconfigurability analysis for piecewise affine systems in discrete-time against actuator faults using state feedback is investigated in [23], where only complete loss of actuators is considered. A control reconfiguration method for PWA continuous-time systems is proposed in [20,21]. In these works, it is assumed that the fault has already been detected and estimated correctly. The aim is to stabilize the faulty closed-loop by inserting a reconfiguration block between the faulty plant and the nominal controller without applying any changes to the nominal controller. A fault detection and identification method for bimodal slab PWL systems with partial loss of actuator gains is proposed in [16]. The constant partial gain loss in actuators is estimated using an observer.

In this work, we consider the problem of fault estimation and active fault tolerant control of multi-modal PWL systems using an observer-based output feedback PWL controller. In contrast to [25] our method in this paper is an active FTC method since we estimate the fault and compensate for its effect. Moreover, in this paper we assume that the controller is output-based since in practice usually full state variables are not available for feedback. In contrast to [16] our work is not limited to bimodal systems. Moreover, Ref. [16] only considers fault detection and estimation while in our work we also address the problem of fault estimation and FTC for PWL systems. The contributions of this paper are as follows:

- First, a robust state and fault estimator for the PWL system is designed that can simultaneously estimate the states of the system and actuator faults effectively. Sufficient conditions in terms of LMIs for ISS of the estimator are given. Moreover, the ISS gains of the system with respect to disturbance are obtained and can be minimized by solving a convex optimization problem with LMI constraints. The proposed method can deal with constant as well as time-varying actuator faults. 
- Second, the estimation of the fault is used to compensate the effect of fault on the input. A PWL state feedback which uses the estimation of the state from the estimator is designed. We provide sufficient conditions in terms of LMIs for the ISS of the controller. Furthermore, the ISS gains of the feedback controller with respect to disturbances and estimation error are obtained which can be minimized by solving a number of optimization problems with LMI constraints.

- Third, we show that separate design of the estimator and the controller yields ISS of the closed-loop system. The closed-loop system remains stable after occurrence of a fault. We obtain the ISS gain for the overall closed-loop system with respect to the disturbance and estimation error and show that this gain could be minimized by minimizing the corresponding gains for the estimator and state feedback controller.

This paper is organized as follows. Notation and preliminaries are given in Section 2. Section 3 gives the system description. In Section 4, the simultaneous fault and state estimator is presented. In Section 5, we present the state feedback controller with the fault compensator. In Section 6, we discuss the design and stability properties of the overall observer-based fault-tolerant control method. Section 7 demonstrates the method a numerical example. Conclusions are given in Section 8.

\section{Preliminaries}

The field of real numbers, the set of nonnegative reals and the set of nonnegative integers are respectively denoted by $\mathbb{R}, \mathbb{R} \geq 0, \mathbb{N}$. For any $x \in \mathbb{R}^{n}, x^{T}$ stands for its transpose and $\|x\|=\sqrt{x^{T} x}$ denotes its Euclidean norm. Also, the $i$-th entry of $x$ is denoted by $x^{i}$. The infinity norm of $x$ denoted by $\|x\|_{\infty}$ is given by $\max _{i}|x|$. Given a sequence $\{v(k)\}_{k \in \mathbb{N}}$, its supremum norm i.e $\sup _{k \in \mathbb{N}}\|v(k)\|$ is denoted by $\|v\|_{\infty}$.

A function $\gamma: \mathbb{R}_{\geq 0} \rightarrow \mathbb{R}_{\geq 0}$ is a class $\mathcal{K}$ function if it is continuous, strictly increasing, and $\gamma(0)=0 . \gamma$ is a class $\mathcal{K}_{\infty}$ function if it is a class $\mathcal{K}$ function and also it satisfies $\gamma(s) \rightarrow \infty$ as $s \rightarrow \infty$. A function $\beta$ is a class $\mathcal{K} \mathcal{L}$ function if for each fixed $k \in \mathbb{R}_{\geq 0}$, the function $\beta(\cdot, k) \in \mathcal{K}$, and for each fixed $s \in \mathbb{R}_{\geq 0}$, the function $\beta(s, \cdot)$ is decreasing and $\beta(s, k) \rightarrow 0$ as $k \rightarrow \infty$. In the following we recall definitions of input-to-state stability for nonlinear discrete-time system [12]. Consider the following nonlinear discrete-time system:

$$
x(k+1)=f(x(k), v(k)),
$$

where $x(k) \in \mathbb{R}^{n}$ is the state, and $v(k) \in \mathbb{R}^{d}$ is an unknown input disturbance.

Definition 1. The nonlinear system (1) is called Input-to-state stable (ISS) with respect to the input $v$ if there exist a $\mathcal{K} \mathcal{L}$-function $\beta$ and a class $\mathcal{K}$-function $\gamma$ such that for each initial condition $x(0) \in \mathbb{R}^{n}$ and all inputs $\{v(k)\}_{k \in \mathbb{N}}$, all solutions of the system satisfy

$$
\|x(k)\| \leq \beta(\|x(0)\|, k)+\gamma\left(\|v\|_{\infty}\right)
$$

The function $\gamma$ is called the ISS gain of Eq. (1) with respect to the input $v$.

Theorem 1 (Jiang and Wang [12], Lazar and Heemels [14]). Let $V: \mathbb{R}^{n} \rightarrow \mathbb{R}_{\geq 0}$ be a continuous function. If there exist a class $\mathcal{K}_{\infty}$ functions $\alpha_{1}$ and $\alpha_{2}$ such that

$$
\alpha_{1}(\|x\|) \leq V(x) \leq \alpha_{2}(\|x\|), \quad \forall x \in \mathbb{R}^{n}
$$


and if there exist a class $\mathcal{K}_{\infty}$ function $\alpha_{3}$ and a $\mathcal{K}$ function $\sigma$ such that

$$
V(f(x, v))-V(x) \leq-\alpha_{3}(\|x\|)+\sigma(\|x\|), \quad \forall x \in \mathbb{R}^{n}, \quad \forall v \in \mathbb{R}^{d},
$$

then, the system (1) is ISS with respect to the input $v$. Furthermore, let $\alpha_{1}(s)=a s^{\lambda}, \alpha_{2}(s)=b s^{\lambda}$, $\alpha_{3}(s)=c s^{\lambda}$, where $a, b, c$ are positive constants with $c \leq b$, then

$$
\beta(s, k)=\alpha_{1}^{-1}\left(2 \rho^{k} \alpha_{2}(s)\right), \gamma(s)=\alpha_{1}^{-1}\left(\frac{2 \sigma(s)}{1-\rho}\right),
$$

where $\rho=1-c / b \in[0,1)$.

A function $V$ that satisfies Eqs. (3) and (4) is called an ISS-Lyapunov function for the system (1).

\section{Piecewise linear systems}

We consider a PWL discrete-time system of the following form:

$$
\begin{aligned}
& x(k+1)=A_{i} x(k)+B_{i} u(k)+E_{i} f(k)+D_{i} w(k), \\
& y(k)=C_{i} x(k) \quad \text { for } y(k) \in \mathcal{R}_{i}, \quad i \in \mathcal{I},
\end{aligned}
$$

where $x(k) \in \mathbb{R}^{n}$ is the state, $u(k) \in \mathbb{R}^{m}$ is the control input, $f(k) \in \mathbb{R}^{r}$ represents the actuator fault, $w(k) \in \mathbb{R}^{d}$ is the disturbance input, $y(k) \in \mathbb{R}^{p}$ is the measured output. $A_{i}, B_{i}, E_{i}, C_{i}$ are constant real matrices with appropriate dimensions. $\left\{\mathcal{R}_{i}\right\}_{i=1}^{s} \subseteq \mathbb{R}^{p}$ denotes a partition of the output space into a number of polyhedral regions $\mathcal{R}_{i}, i \in \mathcal{I}=\{1, \ldots, s\}$. Each polyhedral region is given by

$$
\mathcal{R}_{i}=\left\{y \mid H_{i} y \leq h_{i}\right\} .
$$

All possible switchings from region $\mathcal{R}_{i}$ to $\mathcal{R}_{j}$ are represented by the set $\mathcal{S}$

$$
\mathcal{S}:=\left\{(i, j) \mid y(k) \in \mathcal{R}_{i}, y(k+1) \in \mathcal{R}_{j}\right\} .
$$

\section{Simultaneous state and fault estimation}

In this section, we focus on the estimation of the states of the system and the fault using a PWL observer such that the estimation error converges to zero and at the same time the effect of the disturbance input on the fault estimation error is attenuated. The estimation of the states is later used for design of an observer-based controller and the estimate of the fault is used for compensating the effect of the fault. To simultaneously estimate the fault and the states, the estimate of the fault is augmented to the system as an additional state. Therefore, we have the following PWL augmented fault estimator:

$$
\begin{aligned}
& \hat{x}(k+1)=A_{i} \hat{x}(k)+B_{i} u(k)+E_{i} \hat{f}(k)+L_{i}(y(k)-\hat{y}(k)), \\
& \hat{y}(k)=C_{i} \hat{x}(k) \text { for } y(k) \in \mathcal{R}_{i}, \quad i \in \mathcal{I}, \\
& \hat{f}(k+1)=\hat{f}(k)+F_{i}(y(k)-\hat{y}(k)),
\end{aligned}
$$

where $\hat{x}(k) \in \mathbb{R}^{n}$ is the estimated state, $\hat{y}(k) \in \mathbb{R}^{p}$ is the estimated output, and $\hat{f}(k) \in \mathbb{R}^{r}$ is the estimated fault. $L_{i} \in \mathbb{R}^{n \times p}$ and $F_{i} \in \mathbb{R}^{r \times p}, i=1, \ldots, s$ are observer gains to be designed. The following estimation errors are defined:

$$
e_{x}(k)=x(k)-\hat{x}(k),
$$




$$
e_{f}(k)=f(k)-\hat{f}(k)
$$

then, the dynamic of the state estimation error is governed by

$$
\begin{aligned}
e_{x}(k+1) & =x(k+1)-\hat{x}(k+1)=\left(A_{i}-L_{i} C_{i}\right) e_{x}(k)+E_{i}(f(k)-\hat{f}(k))+D_{i} w(k) \\
& =\left(A_{i}-L_{i} C_{i}\right) e_{x}(k)+E_{i} e_{f}(k)+D_{i} w(k)
\end{aligned}
$$

Also, for the dynamic of the fault estimation error we have

$$
\begin{aligned}
e_{f}(k+1) & =f(k+1)-\hat{f}(k+1)=f(k+1)-\hat{f}(k)-F_{i} C_{i} x(k)+F_{i} C_{i} \hat{x}(k) \\
& =f(k+1)-f(k)+f(k)-\hat{f}(k)-F_{i} C_{i}(x(k)-\hat{x}(k))=e_{f}(k)-F_{i} C_{i} e_{x}(k)+\Delta f(k),
\end{aligned}
$$

where $\Delta f(k)=f(k+1)-f(k)$. Therefore, the dynamics of the error system is given by

$$
\left[\begin{array}{c}
e_{x}(k+1) \\
e_{f}(k+1)
\end{array}\right]=\left[\begin{array}{cc}
A_{i}-L_{i} C_{i} & E_{i} \\
-F_{i} C_{i} & I
\end{array}\right]\left[\begin{array}{c}
e_{x}(k) \\
e_{f}(k)
\end{array}\right]+\left[\begin{array}{cc}
D_{i} & 0 \\
0 & I
\end{array}\right]\left[\begin{array}{c}
w(k) \\
\Delta f(k)
\end{array}\right]
$$

To simplify the notation, let

$$
\begin{aligned}
& \tilde{e}(k)=\left[\begin{array}{l}
e_{x}(k) \\
e_{f}(k)
\end{array}\right], \quad \tilde{A}_{i}=\left[\begin{array}{cc}
A_{i} & E_{i} \\
0 & I
\end{array}\right], \quad \tilde{L}_{i}=\left[\begin{array}{c}
-L_{i} \\
-F_{i}
\end{array}\right], \\
& \tilde{C}_{i}=\left[\begin{array}{ll}
C_{i} & 0
\end{array}\right], \quad \tilde{D}_{i}=\left[\begin{array}{cc}
D_{i} & 0 \\
0 & I
\end{array}\right], \quad v(k)=\left[\begin{array}{c}
w(k) \\
\Delta f(k)
\end{array}\right] .
\end{aligned}
$$

Then, the dynamic of the estimation error is described by

$$
\tilde{e}(k+1)=\left(\tilde{A}_{i}+\tilde{L}_{i} \tilde{C}_{i}\right)+\tilde{D}_{i} v(k), \quad \text { for } y(k) \in \mathcal{R}_{i} .
$$

In this system, both the original disturbance $w$ and the variation of the fault in case of a timevarying fault would affect the system as a disturbance $v$. The goal is to design the observer gains $\tilde{L}_{i}$, such that the system is ISS with respect to the $v$ and to find the corresponding ISS gain.

Theorem 2. If there exist symmetric matrices $P_{i}=P_{i}^{T}>0$, matrices $G_{i}$, and $U_{i}, i=1, \ldots, s$ and a scalar $\sigma_{v} \geq 1$ such that the following set of LMIs are satisfied:

$$
\left[\begin{array}{cccc}
P_{j}-G_{i}-G_{i}^{T} & 0 & G_{i} \tilde{A}_{i}+U_{i} \tilde{C}_{i} & G_{i} \tilde{D}_{i} \\
* & -I & I & 0 \\
* & * & -P_{i} & 0 \\
* & * & * & -\sigma_{v} I
\end{array}\right]<0, \quad \forall i, j \in \mathcal{S},
$$

then, the estimation error system (17) is ISS with respect to $v$ with ISS gain $\gamma_{S}(s)=\sigma_{v}$ s. The observer gains are obtained by

$$
\tilde{L}_{i}=G_{i}^{-1} U_{i} \text {. }
$$

Moreover, $V(\tilde{e}(k))=\tilde{e}(k)^{T} P_{i} \tilde{e}(k), i \in \mathcal{R}_{i}, i \in \mathcal{I}$ is an ISS Lyapunov function which satisfies

$$
\begin{aligned}
& \|\tilde{e}(k)\|^{2} \leq V(\tilde{e}(k)) \leq \sigma_{v}\|\tilde{e}(k)\|^{2}, \\
& V(\tilde{e}(k+1))-V(\tilde{e}(k)) \leq-\|\tilde{e}(k)\|^{2}+\sigma_{v}\|v(k)\|^{2},
\end{aligned}
$$

for all $\tilde{e}(k) \in \mathbb{R}^{n+r}, v \in \mathbb{R}^{d}$. 
Proof. If Eq. (18) is satisfied then, we have

$$
\left[\begin{array}{cc}
-I & I \\
I & -P_{i}
\end{array}\right]<0 \text {. }
$$

Using Schur complement, it implies that $P_{i}>I$. Also, Eq. (18) implies

$$
\left[\begin{array}{cc}
P_{j}-G_{i}-G_{i}^{T} & G_{i} \\
* & -\sigma_{v} I
\end{array}\right]<0 .
$$

This implies that $G_{i}+G_{i}^{T}-P_{j}>\sigma_{\nu} G_{i} G_{i}^{T} \geq 0$. From the fact that $G_{i} P_{j}^{-1} G_{i}^{T} \geq G_{i}+G_{i}^{T}-P_{j}$, it follows that $G_{i} P_{j}^{-1} G_{i}^{T} \geq \sigma_{v}^{-1} G_{i} G_{i}^{T}$. Therefore, $P_{j}<\sigma_{v} I$, and we have $P_{i}>I$ which together result in Eq. (20).

Now to prove Eq. (21), we use the relation $G_{i} P_{j}^{-1} G_{i}^{T} \geq G_{i}+G_{i}^{T}-P_{j}$ again which implies that feasibility of Eq. (18) is a sufficient condition for the feasibility of following matrix inequality

$$
\left[\begin{array}{cccc}
-G_{i} P_{j}^{-1} G_{i}^{T} & 0 & G_{i} \tilde{A}_{i}+U_{i} \tilde{C}_{i} & G_{i} \tilde{D}_{i} \\
* & -I & I & 0 \\
* & * & -P_{i} & 0 \\
* & * & * & -\sigma_{v} I
\end{array}\right]<0 \quad \forall i, j \in \mathcal{S},
$$

Pre- and post-multiplying the above inequality with $\operatorname{diag}\left\{G_{i}^{-1}, I, I, I\right\}$ and its transpose and noting that $\tilde{L}_{i}=G_{i}^{-1} U_{i}$, we get

$$
\left[\begin{array}{cccc}
-P_{j}^{-1} & 0 & \tilde{A}_{i}+\tilde{L}_{i} \tilde{C}_{i} & \tilde{D}_{i} \\
* & -I & I & 0 \\
* & * & -P_{i} & 0 \\
* & * & * & -\sigma_{v} I
\end{array}\right]<0 \quad \forall i, j \in \mathcal{S},
$$

Applying the Schur complement to the above matrix inequality yields

$$
\left[\begin{array}{cc}
-P_{i} & 0 \\
0 & -\sigma_{v} I
\end{array}\right]-\left[\begin{array}{cc}
\left(\tilde{A}_{i}+\tilde{L}_{i} \tilde{C}_{i}\right)^{T} & I \\
\tilde{D}_{i}^{T} & \\
0 & I
\end{array}\right]\left[\begin{array}{cc}
-P_{j} & 0 \\
0 & -I
\end{array}\right]\left[\begin{array}{cc}
\tilde{A}_{i}+\tilde{L}_{i} \tilde{C}_{i} & \tilde{D}_{i} \\
I & 0
\end{array}\right]<0,
$$

which is equal to

$$
\left[\begin{array}{cc}
\left(\tilde{A}_{i}+\tilde{L}_{i} \tilde{C}_{i}\right)^{T} P_{j}\left(\tilde{A}_{i}+\tilde{L}_{i} \tilde{C}_{i}\right)-P_{i}+I & \left(\tilde{A}_{i}+\tilde{L}_{i} \tilde{C}_{i}\right)^{T} P_{j} \tilde{D}_{i} \\
* & \tilde{D}_{i} P_{j} \tilde{D}_{i}-\sigma_{v} I
\end{array}\right]<0
$$

Pre- and post-multiplying the above inequality with $\left[\tilde{e}(k)^{T} v(k)^{T}\right]^{T}$ and its transpose gives

$$
\begin{aligned}
& \left(\left(\tilde{A}_{i}+\tilde{L}_{i} \tilde{C}_{i}\right) \tilde{e}(k)+\tilde{D}_{i} v(k)\right)^{T} P_{j}\left(\left(\tilde{A}_{i}+\tilde{L}_{i} \tilde{C}_{i}\right) \tilde{e}(k)+\tilde{D}_{i} v(k)\right)-\tilde{e}(k)^{T} P_{i} \tilde{e}(k) \\
& \leq-\tilde{e}(k)^{T} \tilde{e}(k)+\sigma_{\nu} v(k)^{T} v(k) .
\end{aligned}
$$

Since $\tilde{e}(k+1)=\left(\tilde{A}_{i}+\tilde{L}_{i} \tilde{C}_{i}\right) \tilde{e}(k)+\tilde{D}_{i} v(k)$, the above inequality can be rewritten as

$$
(\tilde{e}(k+1))^{T} P_{j}(\tilde{e}(k+1))-\tilde{e}(k)^{T} P_{i} \tilde{e}(k) \leq-\tilde{e}(k)^{T} \tilde{e}(k)+\sigma_{v} v(k)^{T} v(k) .
$$

which is equal to Eq. (21). Therefore, based on Theorem 1, the error system is ISS with respect to $v$. Now, we move to the calculation of the ISS gain. The inequality (21) together with Eq. (20) 
gives

$$
V(\tilde{e}(k+1)) \leq V(\tilde{e}(k))\left(1-\frac{1}{\sigma_{v}}\right)+\sigma_{v}\|v(k)\|^{2} .
$$

Applying Eq. (30) for $k=0$ up to $k=k$ in an inductive manner gives

$$
\begin{aligned}
V(\tilde{e}(k)) & \leq V(\tilde{e}(0))\left(1-\frac{1}{\sigma_{v}}\right)^{k}+\sigma_{v} \sum_{l=0}^{k-1}\left(1-\frac{1}{\sigma_{v}}\right)^{k-l-1}\|v(l)\|^{2} \\
& \leq\left(1-\frac{1}{\sigma_{v}}\right)^{k} V(\tilde{e}(0))+\sigma_{v}^{2}\|v(l)\|_{\infty}^{2} .
\end{aligned}
$$

Since from Eq. (20) $V(\tilde{e}(0)) \leq \sigma_{v}\|\tilde{e}(0)\|^{2}$, the above inequality implies

$$
\|\tilde{e}(k)\| \leq \sqrt{\sigma_{v}}\left(1-\frac{1}{\sigma_{v}}\right)^{k / 2}\|\tilde{e}(0)\|+\sigma_{v}\|v\|_{\infty} .
$$

This proves that the system is ISS with respect to $v$ with the ISS gain $\gamma_{S}(s)=\sigma_{v} s, s \in \mathbb{R} \geq 0$.

To minimize the ISS gain, the following optimization problem is solved.

$$
\min _{P_{i}, G_{i}, U_{i}} \sigma_{v}
$$

$$
\text { s.t. } \quad \text { Eq. (18) }
$$

The above optimization problem is a convex optimization problem with a set of LMI constraints which can be solved efficiently using available softwares such as YALMIP/SeDuMi or YALMIP/LMILAB [15].

\section{Fault accommodation}

The state estimation is used by a PWL feedback control to stabilize the system and the estimate of the fault is used to compensate for the effect of the fault by adding a correction term to the input of the system to generate the desired control input that stabilize the faulty system. Before proceeding, we state the following assumption.

Assumption 1. $\operatorname{rank}\left(B_{i}, E_{i}\right)=\operatorname{rank}\left(B_{i}\right)$

Lemma 1 (Jiang et al. [10]). Under Assumption1, there exist a matrix $B_{i}^{*} \in \mathbb{R}^{m \times n}$ such that

$$
\left(I-B_{i} B_{i}^{*}\right) E_{i}=0 .
$$

To stabilize the faulty system, the following PWL output feedback strategy with fault compensator is introduced:

$$
u(k)=K_{i} \hat{x}(k)-B_{i}^{*} E_{i} \hat{f}(k) \text { for } y(k) \in \mathcal{R}_{i}, \quad i \in \mathcal{I},
$$

where the term $B_{i}^{*} E_{i} \hat{f}(k)$ is added to the input to compensate for the effect of the fault. Eqs. (35) and (6) together with $\hat{x}(k)=x(k)-e_{x}(k)$ imply

$$
\begin{aligned}
x(k+1) & =A_{i} x(k)+B_{i} u(k)+E_{i} f(k)+D_{i} w(k) \\
& =A_{i} x(k)+B_{i} K_{i} \hat{x}(k)-B_{i} B_{i}^{*} E_{i} \hat{f}(k)+E_{i} f(k)+D_{i} w(k) \\
& =\left(A_{i}+B_{i} K_{i}\right) x(k)-B_{i} K_{i} e_{x}(k)-B_{i} B_{i}^{*} E_{i} \hat{f}(k)+E_{i} f(k)+D_{i} w(k) \\
& =\bar{A}_{i} x(k)-B_{i} B_{i}^{*} E_{i} \hat{f}(k)+E_{i} f(k)+E_{i} \hat{f}(k)-E_{i} \hat{f}(k)+D_{i} w(k)-B_{i} K_{i} e_{x}(k) \\
& =\bar{A}_{i} x(k)+\left(I-B_{i} B_{i}^{*}\right) E_{i} \hat{f}(k)+E_{i} e_{f}(k)+D_{i} w(k)-B_{i} K_{i} e_{x}(k)
\end{aligned}
$$




$$
=\bar{A}_{i} x(k)+\left[\begin{array}{ll}
D_{i} & E_{i}
\end{array}\right]\left[\begin{array}{c}
w(k) \\
e_{f}(k)
\end{array}\right]-B_{i} K_{i} e_{x}(k),
$$

where $\bar{A}_{i}=A_{i}+B_{i} K_{i}$. Denote

$$
\bar{D}_{i}=\left[\begin{array}{ll}
D_{i} & E_{i}
\end{array}\right], \quad \bar{w}(k)=\left[\begin{array}{c}
w(k) \\
e_{f}(k)
\end{array}\right] .
$$

Then, the dynamics of the faulty system with fault compensator is given by

$$
x(k+1)=\bar{A}_{i} x(k)+\bar{D}_{i} \bar{w}(k)-B_{i} K_{i} e_{x}(k), \quad \text { for } y(k) \in \mathcal{R}_{i}, \quad i \in \mathcal{I} .
$$

In the following we investigate the ISS of the above system.

Theorem 3. If there exist symmetric matrices $Q_{i}=Q_{i}^{T}$ and matrices $Y_{i}$ for $i \in \mathcal{I}$ and scalars $\sigma_{x w} \geq 1$ and $\eta>0$ such that

$$
\begin{aligned}
& {\left[\begin{array}{ccccc}
-Q_{j} & 0 & A_{i} Q_{i}+B_{i} Y_{i} & \bar{D}_{i} & -B_{i} Y_{i} \\
0 & -I & Q_{i} & 0 & 0 \\
* & * & -Q_{i} & 0 & 0 \\
* & * & * & -\sigma_{x w} I & 0 \\
* & * & * & * & -\sigma_{x w} I
\end{array}\right]<0 \quad \forall(i, j) \in \mathcal{S},} \\
& Q_{i} \geq \eta I, \quad \forall i \in \mathcal{I},
\end{aligned}
$$

then, the closed-loop system (38) is ISS with respect to $w, e_{f}$, and $e_{x}$ with ISS gain $\gamma_{K}(s)=\sqrt{\eta^{-3} \sigma_{x w}}$. The controller gains $K_{i}$ are given by

$$
K_{i}=Y_{i} Q_{i}^{-1} \text {. }
$$

Moreover, $V(x(k))=x(k)^{T} P_{i} x(k), i \in \mathcal{R}_{i}, i \in \mathcal{I}$ with $P_{i}=Q_{i}^{-1}$ is an ISS Lyapunov function for the closed-loop system (38) which satisfies

$$
\begin{aligned}
& \|x(k)\|^{2} \leq V(x(k)) \leq \eta^{-1}\|x(k)\|^{2}, \\
& V(x(k+1))-V(x(k)) \leq-\|x(k)\|^{2}+\eta^{-2} \sigma_{x w}\left\|\left[\begin{array}{c}
\bar{w}(k) \\
e_{x}(k)
\end{array}\right]\right\|^{2},
\end{aligned}
$$

for all $x(k) \in \mathbb{R}^{n},\left[w(k)^{T} e_{f}(k)^{T} e_{x}(k)^{T}\right]^{T} \in \mathbb{R}^{d+r+n}$.

Proof. If Eq. (39) is feasible, then it holds that

$$
\left[\begin{array}{cc}
-I & Q_{i} \\
* & -Q_{i}
\end{array}\right]<0,
$$

which by using Schur complement implies that $Q_{i}^{-1}>I$. Also, feasibility of Eq. (40) implies that $Q_{i}^{-1} \leq \eta^{-1} I$. Therefore, feasibility of Eqs. (39) and (40) implies Eq. (42). Note that $I \leq Q_{i}^{-1} \leq \eta^{-1} I$ implies that $\eta<1$. Post- and pre-multiplying Eq. (39) by $\operatorname{diag}\left\{I, I, Q_{i}^{-1}, I, Q_{i}^{-1}\right\}$ 
and its transpose and using the relation $K_{i}=Y_{i} Q_{i}^{-1}$ we get

$$
\left[\begin{array}{ccccc}
-Q_{j} & 0 & A_{i}+B_{i} K_{i} & \bar{D}_{i} & -B_{i} K_{i} \\
0 & -I & I & 0 & 0 \\
* & * & -Q_{i}^{-1} & 0 & 0 \\
* & * & * & -\sigma_{x w} I & 0 \\
* & * & * & * & -\sigma_{x w} Q_{i}^{-2}
\end{array}\right]<0 \quad \forall(i, j) \in \mathcal{S},
$$

From Eq. (40), we have that: $Q_{i}^{-2} \leq \eta^{-2} I$. Therefore, if the above matrix inequality is feasible, it implies that the following inequality is also feasible:

$$
\left[\begin{array}{ccccc}
-Q_{j} & 0 & A_{i}+B_{i} K_{i} & \bar{D}_{i} & -B_{i} K_{i} \\
0 & -I & I & 0 & 0 \\
* & * & -Q_{i}^{-1} & 0 & 0 \\
* & * & * & -\sigma_{x w} I & 0 \\
* & * & * & * & -\eta^{-2} \sigma_{x w} I
\end{array}\right]<0 \quad \forall(i, j) \in \mathcal{S},
$$

Using Schur Complement we get

$$
\begin{aligned}
& {\left[\begin{array}{ccc}
Q_{i}^{-1}+I & 0 & 0 \\
* & -\sigma_{x w} I & 0 \\
* & * & -\eta^{-2} \sigma_{x w} I
\end{array}\right]} \\
& +\left[\left(A_{i}+B_{i} K_{i}\right)^{T} \bar{D}_{i}^{T}-\left(B_{i} K_{i}\right)^{T}\right]^{T}\left[Q_{j}^{-1}\right]\left[A_{i}+B_{i} K_{i} \bar{D}_{i}-B_{i} K_{i}\right]<0
\end{aligned}
$$

Pre- and post-multiplying the above inequality with $\left[x(k)^{T} \bar{w}(k)^{T} e(k)^{T}\right]^{T}$ and its transpose and knowing that $x(k+1)=\left(A_{i}+B_{i} K_{i}\right) x(k)+\bar{D}_{i} \bar{w}(k)-B_{i} K_{i} e(k)$ we get

$$
\begin{aligned}
& x(k+1)^{T} Q_{j}^{-1} x(k+1)-x(k) Q_{i}^{-1} x(k) \\
& \leq-x(k)^{T} x(k)+\sigma_{x w} \bar{w}(k)^{T} \bar{w}(k)+\eta^{-2} \sigma_{x w} e(k)^{T} e(k)
\end{aligned}
$$

Since $\eta \leq 1$, it is true that $\eta^{-2} \sigma_{x w} \geq \sigma_{x w}$. Therefore, we have

$$
x(k+1)^{T} Q_{j}^{-1} x(k+1)-x(k) Q_{i}^{-1} x(k) \leq \eta^{-2} \sigma_{x w}\left[\bar{w}(k)^{T} e_{x}(k)^{T}\right]\left[\begin{array}{c}
\bar{w}(k) \\
e_{x}(k)^{T}
\end{array}\right],
$$

which is equal to Eq. (43). Therefore, based on the Theorem 1, the closed-loop system is ISS with respect to $\bar{w}(k)$ and $e_{x}(k)$. The ISS gain is calculated using the same procedure as we used for the estimation error system. In this case $\gamma_{K}(s)=\sqrt{\eta^{-3} \sigma_{x w}} s$. $\square$

To minimize the ISS gain for the state feedback controller, the following optimization problem must be solved.

$$
\begin{array}{ll}
\min _{Q_{i}, Y_{i}} & \sqrt{\eta^{-3} \sigma_{x w}} \\
\text { s.t. } & \left\{\begin{array}{l}
\text { Eq. (39) } \\
\text { Eq. (40) }
\end{array}\right.
\end{array}
$$


The above optimization problem is not convex, but it can be efficiently solved by performing a line search in $\eta$ and minimizing for $\sigma_{x w}$.

\section{Observer-based fault accommodation}

In this section, we show that if we design the controller and the fault estimator separately based on the conditions provided in the previous sections, then the overall closed-loop system which consists of the plant, the estimator, and the controller is stable after occurrence of a fault.

The dynamics of the closed-loop systems is given by

$$
\left[\begin{array}{c}
x(k+1) \\
\tilde{e}(k+1)
\end{array}\right]=\left[\begin{array}{cc}
A_{i}+B_{i} K_{i} & {\left[E_{i}-B_{i} K_{i}\right.} \\
0 & \tilde{A}_{i}+\tilde{L}_{i} \tilde{C}_{i}
\end{array}\right]\left[\begin{array}{c}
x(k+1) \\
\tilde{e}(k+1)
\end{array}\right]+\left[\begin{array}{cc}
D_{i} & 0 \\
D_{i} & 0 \\
0 & I
\end{array}\right]\left[\begin{array}{c}
w(k) \\
\Delta f(k)
\end{array}\right] .
$$

Theorem 4. If there exists an estimator that satisfies the hypotheses of Theorem 2 and there exists a state feedback controller that satisfied the hypotheses of Theorem3, then the closed-loop system (51) is ISS with respect to $\left[\begin{array}{c}w(k) \\ \Delta f(k)\end{array}\right]$ before and after a fault event at time $k_{f}$.

Proof. We consider the following candidate Lyapunov function

$$
V_{\mu}(x(k), \tilde{e}(k))=V_{x}(x(k))+\mu V_{e}(\tilde{e}(k)),
$$

with $\mu>0$ for the closed-loop system. From Eqs. (21) and (43), we have

$$
\begin{aligned}
V_{\mu}(x(k+1), \tilde{e}(k+1))-V_{\mu}(x(k), \tilde{e}(k)) \leq-\|x(k)\|^{2}+\sigma_{x w}\|\bar{w}(k)\|^{2}+\eta^{-2} \sigma_{x w}\left\|e_{x}(k)\right\|^{2} \\
\quad-\mu\|\tilde{e}(k)\|^{2}+\mu \sigma_{v}\|w(k)\|^{2}+\mu \sigma_{v}\|\Delta f(k)\|^{2} \\
\leq-\|x(k)\|^{2}+\sigma_{x w}\|w(k)\|^{2}+\sigma_{x w}\left\|e_{f}(k)\right\|^{2}+\eta^{-2} \sigma_{x w}\left\|e_{x}(k)\right\|^{2} \\
\quad-\mu\left\|e_{x}(k)\right\|^{2}-\mu \sigma_{v}\left\|e_{f}(k)\right\|^{2}+\mu \sigma_{v}\|w(k)\|^{2}+\mu \sigma_{v}\|\Delta f(k)\|^{2} \\
\leq-\|x(k)\|^{2}+\left(\eta^{-2} \sigma_{x w}-\mu\right)\left\|e_{x}(k)\right\|^{2}+\left(\sigma_{x w}-\mu\right)\left\|e_{f}(k)\right\|^{2} \\
\quad+\left(\sigma_{x w}+\mu \sigma_{v}\right)\|w(k)\|^{2}+\mu \sigma_{v}\|\Delta f(k)\|^{2} .
\end{aligned}
$$

If $\mu \geq \eta^{-2} \sigma_{x w}+1$, then we have that

$$
\begin{aligned}
& V_{\mu}(x(k+1), \tilde{e}(k+1))-V_{\mu}(x(k), \tilde{e}(k)) \\
& \quad \leq-\|x(k)\|^{2}-\left\|e_{x}(k)\right\|^{2}-\left\|e_{f}(k)\right\|^{2}+\left(\sigma_{x w}+\mu \sigma_{v}\right)\|w(k)\|^{2}+\mu \sigma_{v}\|\Delta f(k)\|^{2} .
\end{aligned}
$$

Since $\left\|\left[\begin{array}{ll}x^{T} & \tilde{e}^{T}\end{array}\right]^{T}\right\|^{2}=\|x\|^{2}+\|\tilde{e}\|^{2}$, we have

$$
V_{\mu}(x(k+1), \tilde{e}(k+1))-V_{\mu}(x(k), \tilde{e}(k)) \leq-\left\|\left[\begin{array}{c}
x(k) \\
\tilde{e}(k)
\end{array}\right]\right\|^{2}+\left(\sigma_{x w}+\mu \sigma_{v}\right)\|w(k)\|^{2}+\mu \sigma_{v}\|\Delta f(k)\|^{2} .
$$

Moreover,

$$
\|w(k)\|^{2} \leq\left\|\left[w(k)^{T} \quad \Delta f(k)^{T}\right]^{T}\right\|,
$$

and likewise

$$
\|\Delta f(k)\|^{2} \leq\left\|\left[w(k)^{T} \quad \Delta f(k)^{T}\right]^{T}\right\| .
$$

Therefore, it is concluded that

$$
V_{\mu}(x(k+1), \tilde{e}(k+1))-V_{\mu}(x(k), \tilde{e}(k))
$$




$$
\leq-\left\|\left[\begin{array}{c}
x(k) \\
\tilde{e}(k)
\end{array}\right]\right\|^{2}+2\left(\sigma_{x w}+\mu \sigma_{v}\right)\left\|\left[\begin{array}{c}
w(k) \\
\Delta f(k)
\end{array}\right]\right\|^{2}
$$

Also, since $\mu>1$, we have that

$$
\left\|\left[\begin{array}{c}
x(k) \\
\tilde{e}(k)
\end{array}\right]\right\|^{2} \leq\|x(k)\|^{2}+\|\tilde{e}(k)\| \leq\|x(k)\|^{2}+\mu\|\tilde{e}(k)\|^{2} \leq V_{\mu}(x(k), \tilde{e}(k))
$$

Furthermore,

$$
\begin{aligned}
& V_{\mu}(x(k), \tilde{e}(k)) \leq \eta^{-1}\|x(k)\|^{2}+\mu \sigma_{v}\|\tilde{e}(k)\|^{2} \\
& \quad \leq \max \left(\eta^{-1}, \mu \sigma_{v}\right)\|x(k)\|^{2}+\max \left(\eta^{-1}, \mu \sigma_{v}\right)\|e(k)\|^{2} \leq \max \left(\eta^{-1}, \mu \sigma_{v}\right)\left\|\left[\begin{array}{c}
x(k) \\
\tilde{e}(k)
\end{array}\right]\right\|^{2}
\end{aligned}
$$

Because $\mu \geq \eta^{-2} \sigma_{x w}+1$, and $\eta^{-1} \geq 1, \sigma_{x w} \geq 1$ it holds that $\mu \sigma_{v} \geq \eta^{-1}$ which means $\max \left(\eta^{-1}, \mu \sigma_{v}\right)=\mu \sigma_{v}$. Therefore, we have that

$$
\left\|\left[\begin{array}{c}
x(k) \\
\tilde{e}(k)
\end{array}\right]\right\|^{2} \leq V_{\mu}(x(k), \tilde{e}(k)) \leq \mu \sigma_{v}\left\|\left[\begin{array}{c}
x(k) \\
\tilde{e}(k)
\end{array}\right]\right\|^{2} .
$$

Using Theorem 1 with the above inequality together with Eq. (6), it is concluded that the closedloop system is ISS with respect to $w(k)$ and $\Delta f(k)$. Using Eq. (5), the functions $\beta$ and $\gamma$ are calculated as

$$
\begin{aligned}
& \beta(s, k)=\sqrt{2}\left(1-\frac{1}{\mu \sigma_{v}}\right)^{k / 2} \sqrt{\mu \sigma_{v}} s \\
& \gamma(s)=2 \sqrt{\mu \sigma_{v}\left(\sigma_{x w}+\mu \sigma_{v}\right) s} \quad \square
\end{aligned}
$$

It is of interest to minimize the ISS gain. To minimize the ISS gain, we have to find the minimum of the function $f\left(\mu, \sigma_{x w}, \sigma_{v}\right)=\sqrt{\mu \sigma_{v}\left(\sigma_{x w}+\mu \sigma_{v}\right)}$. It is clear that to minimize the function, $\mu, \sigma_{x w}, \sigma_{v}$ must be minimized. Minimum of $\mu$ is obtained when it is chosen as $\eta^{-2} \sigma_{x w}+1$. Therefore, the minimization of gain could obtained by separately minimizing $\eta^{-2} \sigma_{x w}, \sigma_{v}$.

\section{Illustrative example}

We consider the following PWL system which is an extension of the example in [1] by considering the given partition and introducing actuator fault. The system consists of the following four modes:

$$
\begin{aligned}
& A_{1}=\left[\begin{array}{lll}
0.7786 & 0.9908 & 0.1270 \\
0.1616 & 0.8443 & 0.8144 \\
0.9214 & 0.9747 & 0.7825
\end{array}\right], A_{2}=\left[\begin{array}{lll}
0.3984 & 0.3263 & 0.7764 \\
0.7806 & 0.9886 & 0.1297 \\
0.8814 & 0.4718 & 0.3110
\end{array}\right], \\
& A_{3}=\left[\begin{array}{lll}
0.3049 & 0.4247 & 0.8979 \\
0.8448 & 0.2485 & 0.6921 \\
0.7558 & 0.9160 & 0.3636
\end{array}\right], A_{4}=\left[\begin{array}{lll}
0.1194 & 0.3964 & 0.2454 \\
0.1034 & 0.2515 & 0.4983 \\
0.6981 & 0.8655 & 0.2403
\end{array}\right],
\end{aligned}
$$




$$
\begin{aligned}
& B_{1}=\left[\begin{array}{cc}
0.2485 & 0.7409 \\
0.2501 & 0.5257 \\
0 & 0
\end{array}\right], \quad B_{2}=\left[\begin{array}{cc}
0.2722 & 0.6055 \\
0.1576 & 0.1580 \\
0 & 0
\end{array}\right] \text {, } \\
& B_{3}=\left[\begin{array}{cc}
0.4945 & 0.3020 \\
0.9237 & 0.9118 \\
0 & 0
\end{array}\right], \quad B_{4}=\left[\begin{array}{cc}
0.9894 & 0.7205 \\
0.1709 & 0.1519 \\
0 & 0
\end{array}\right] \text {, } \\
& C_{1}=\left[\begin{array}{lll}
0.3815 & 0.6916 & 0.7183
\end{array}\right], \quad C_{2}=\left[\begin{array}{lll}
0.0591 & 0.8258 & 0.4354
\end{array}\right], \\
& C_{3}=\left[\begin{array}{lll}
0.5204 & 0.8010 & 0.9708
\end{array}\right], \quad C_{4}=\left[\begin{array}{llll}
0.6995 & 0.3081 & 0.8767
\end{array}\right], \\
& D_{1}=\left[\begin{array}{l}
0.05 \\
0.05 \\
0.05
\end{array}\right], \quad D_{2}=D_{3}=D_{4}=D_{1} \text {. }
\end{aligned}
$$

The regions $\mathcal{R}_{i}$ are given as

$$
\begin{aligned}
& \mathcal{R}_{1}=\{y \mid 0 \leq y \leq 1\}, \quad \mathcal{R}_{2}=\{y \mid-1 \leq y \leq 0\}, \\
& \mathcal{R}_{3}=\{y \mid y<-1\}, \quad \mathcal{R}_{4}=\{y \mid 1<y\} .
\end{aligned}
$$

We mention that all the matrices $A_{1}, \ldots, A_{4}$ are unstable. We consider both constant and time varying faults. It is assumed that the fault only affects the first input channel, therefore we have

$$
E_{1}=\left[\begin{array}{c}
0.2485 \\
0.2501 \\
0
\end{array}\right], \quad E_{2}=\left[\begin{array}{c}
0.2722 \\
0.1576 \\
0
\end{array}\right], \quad E_{3}=\left[\begin{array}{c}
0.4945 \\
0.9237 \\
0
\end{array}\right], \quad E_{4}=\left[\begin{array}{c}
0.9894 \\
0.1709 \\
0
\end{array}\right] .
$$

It is obvious that $\operatorname{rank}\left(B_{i}, E_{i}\right)=\operatorname{rank}\left(B_{i}\right)$ and therefore Assumption 1 is satisfied. The state and fault estimator is obtained using the optimization problem (33). The minimum value for $\sigma_{v}$ is obtained as 10.99 and the observer gains are given by

$$
L_{1}=\left[\begin{array}{l}
-1.9079 \\
-1.4416 \\
-1.6296
\end{array}\right], \quad L_{2}=\left[\begin{array}{l}
-1.1845 \\
-1.9045 \\
-1.1197
\end{array}\right], \quad L_{3}=\left[\begin{array}{l}
-1.2281 \\
-1.8913 \\
-1.1280
\end{array}\right], \quad L_{4}=\left[\begin{array}{l}
-1.1555 \\
-1.5605 \\
-1.4480
\end{array}\right],
$$

and

$$
F_{1}=-1.6767, \quad F_{2}=-1.8221, \quad F_{3}=-1.2807, \quad F_{4}=-1.1898 .
$$

To design the controller we use Theorem 3. Fig. 1 shows how the ISS gain for the controller changes with respect to the parameter $\eta$. The minimum ISS gain is obtained with $\eta=0.25$. For values of $\eta$ less than 0.25 the LMIs are not feasible. The following gains of the controllers are obtained:

$$
\begin{aligned}
& K_{1}=\left[\begin{array}{ccc}
4.8065 & -2.2163 & -10.1497 \\
-3.0243 & -0.9664 & 2.9298
\end{array}\right], \quad K_{2}=\left[\begin{array}{ccc}
-8.9979 & -10.8573 & 0.4423 \\
3.0291 & 4.1599 & -1.5940
\end{array}\right], \\
& K_{3}=\left[\begin{array}{ccc}
-0.5384 & -2.3396 & -3.7325 \\
-0.5699 & 1.8380 & 2.9377
\end{array}\right], \quad K_{4}=\left[\begin{array}{ccc}
3.3276 & 5.9447 & 12.1102 \\
-4.9775 & -9.0021 & -17.0469
\end{array}\right] .
\end{aligned}
$$


To minimize the gain of the overall closed-loop system $\eta^{-2} \sigma_{x w}$ must be minimized which follows a similar pattern as shown in Fig. 2. Therefore, the minimum ISS gain of the overall closed-loop system is obtained using Eq. (60) as 948.

We consider two different faults. In the first case, we consider the following constant fault:

$$
f_{1}(k)=\left\{\begin{array}{ll}
0 & k \leq 50 \\
2 & k>50
\end{array}\right. \text {. }
$$

Fig. 3 shows the closed-loop output of the system when the fault is not compensated using the estimation of the fault. As it can be seen the origin of the faulty system is not ISS. Using the proposed method, the effect of fault is compensated by adding an appropriate term to the input of the system. The states of the system with fault compensation are depicted in Fig. 4. By compensating the effect of fault, the origin of the closed-loop system is ISS. Fig. 5 shows the simulation result for estimating the fault using the gains obtained as explained above and Fig. 6 shows the states and their estimation. As it can be seen both the fault and states are estimated efficiently.

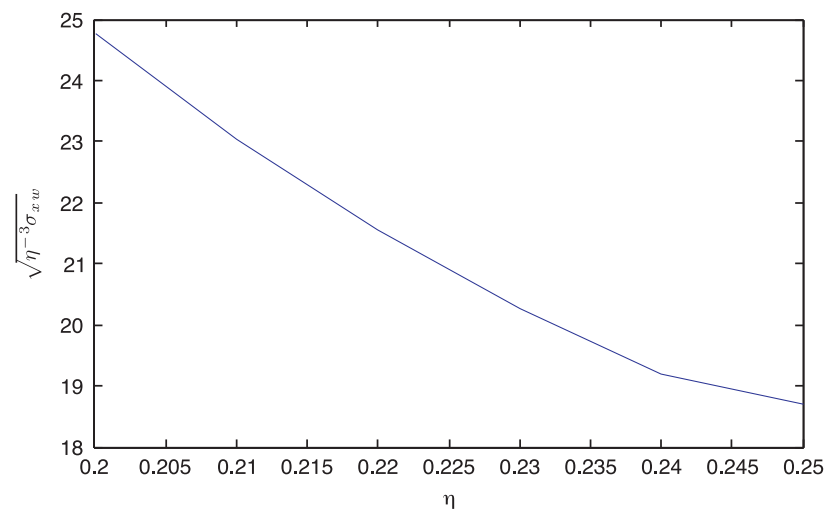

Fig. 1. ISS gain for the controller for a line search in $\eta$.

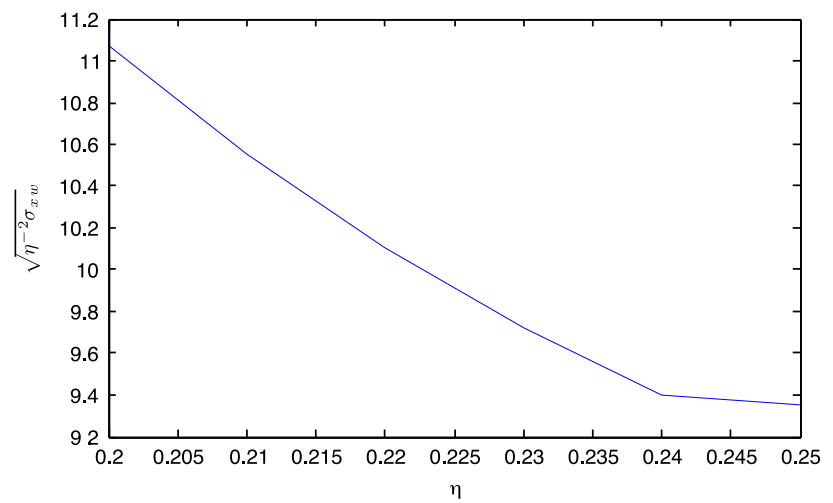

Fig. 2. Minimum values of $\sqrt{\eta^{-2} \sigma_{x w}}$ for a line search in $\eta$. 

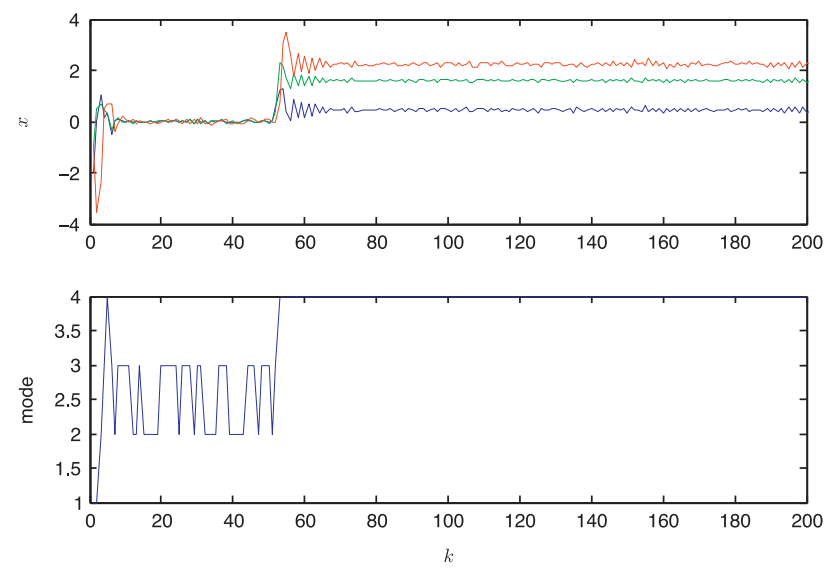

Fig. 3. Top: states of the system without compensating for the fault, Bottom: active mode of the system.
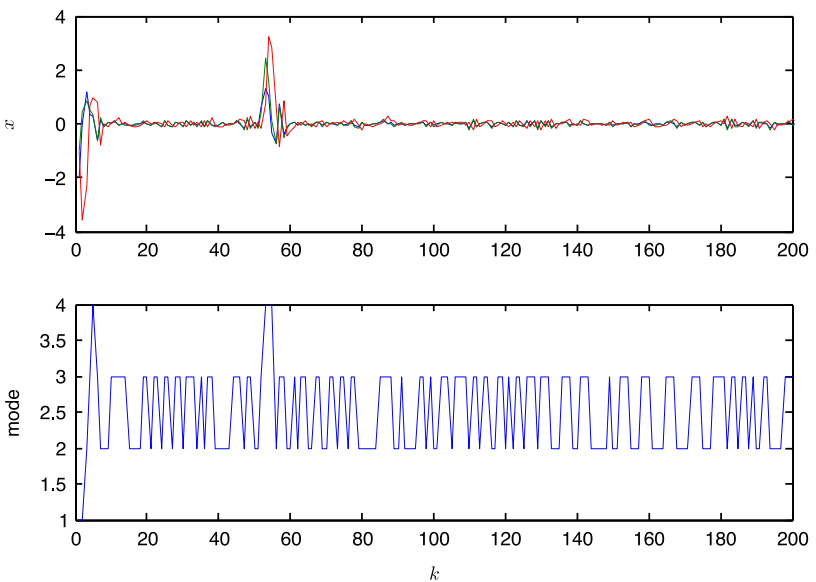

Fig. 4. Top: states of the system with fault compensation, Bottom: active mode of the system.

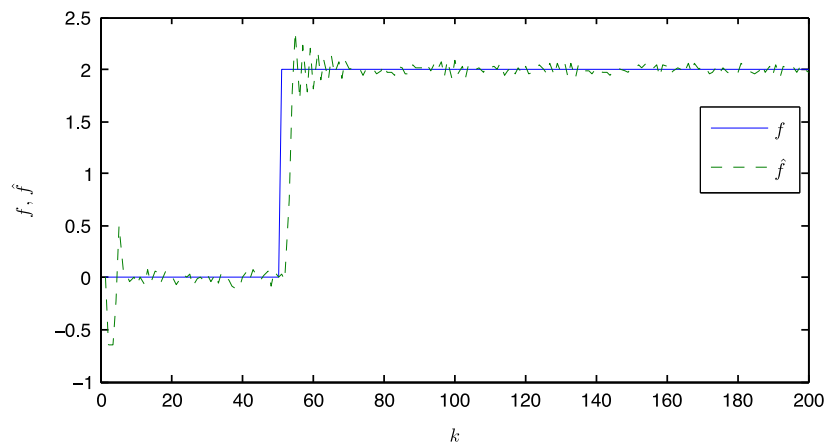

Fig. 5. Fault $f$ and its estimate $\hat{f}$. 

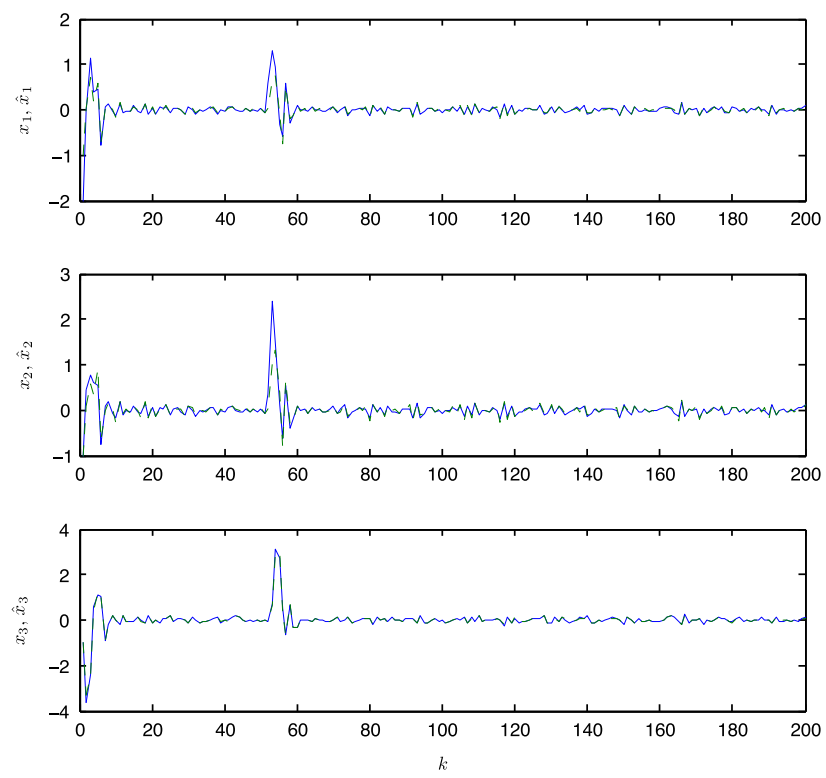

Fig. 6. States of the system (solid lines) and their estimates (dashed lines).

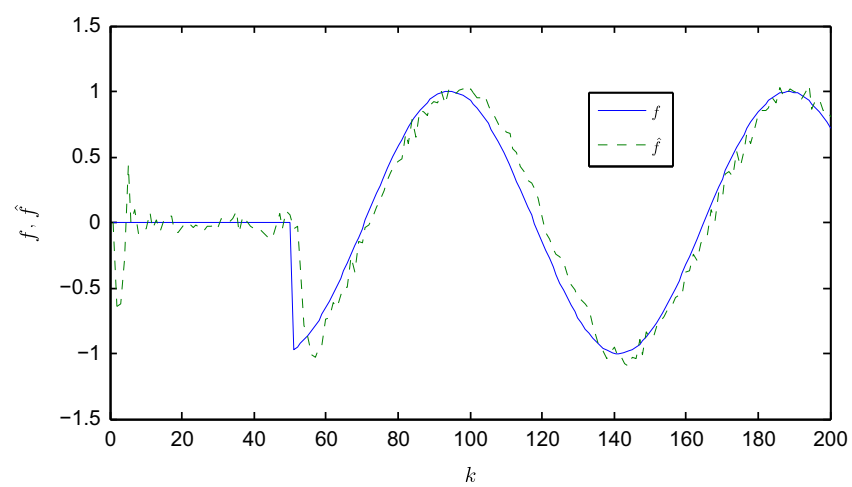

Fig. 7. Fault $f$ and its estimate $\hat{f}$.

For the second scenario we consider a time varying fault

$$
f(k)=\left\{\begin{array}{l}
0 k \leq 50 \\
\cos (k / 15) k>50
\end{array}\right.
$$

Fig. 7 shows the fault and its estimate. Figs. 8 and 9 show the states and mode of the system without and with fault compensation. As the simulation results show, a time-varying fault is also estimated efficiently and then compensated. In the case of time-varying fault variation of fault $\Delta f(k)$ would act as a disturbance for both fault estimation and compensation. As a result the performance of the method depends on the rate of variation of the fault. Based on our simulations if the fault is changing fast, then there would be a considerable error in the estimation. This error would act as a disturbance for fault compensation with degrades the performance of the system. 

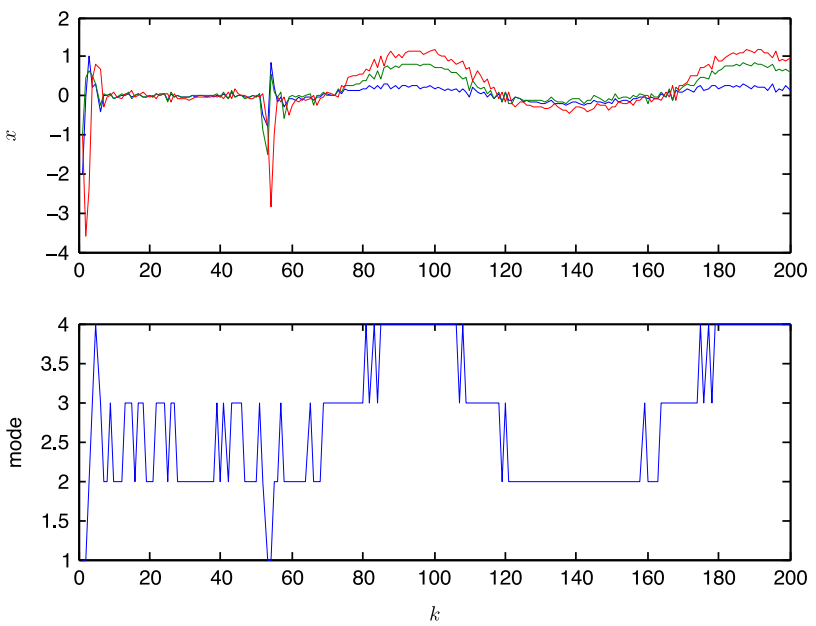

Fig. 8. Top: states of the system, $x$ without compensating for the fault, Bottom: active mode of the system.
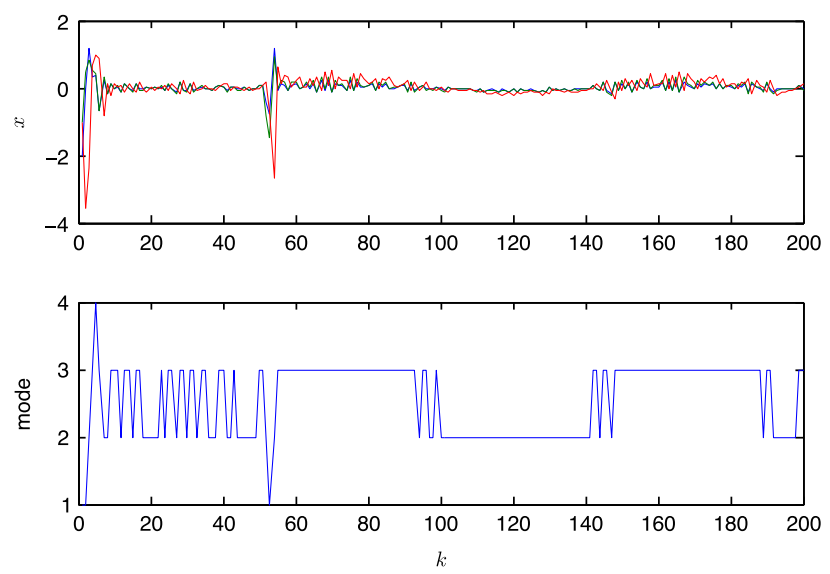

Fig. 9. Top: states of the system, $x$ with fault compensation, Bottom: active mode of the system.

\section{Conclusion}

The problem of fault estimation and fault tolerant control for discrete-time piecewise linear systems is considered in this paper. An observer based active fault tolerant control strategy against actuator faults is proposed in this paper. An observer is designed such that it estimates simultaneously the state of the system and the actuator fault. The estimate of the fault is then used to compensate for the effect of fault by adding a term to the output of a PWL feedback controller. As a result the closed-loop system is an observer based active fault tolerant controller which stabilizes the system in the sense of input-to-state stability. We provide sufficient conditions for ISS of the PWL estimator and feedback controller in terms of LMI conditions. We show that these ISS gains could be minimized by solving a convex optimization problem with LMI constrains. Then, we show that separate design of the estimator and feedback controller guarantees the ISS of the 
overall closed-loop system. The ISS gain for the overall closed-loop systems is obtained in terms of the ISS gains of the estimator and feedback controller and we discuss how to minimize the ISS gain of the overall closed-loop system. Finally the efficiency of the algorithm with respect to both constant and time-varying fault is demonstrated using a numerical example. Future direction of this research is to include the problem of sensor fault reconstruction and compensation.

\section{References}

[1] G.M. Bara, M. Boutayeb, Switched output feedback stabilization of discrete-time switched systems, in: 45th IEEE Conference Decision and Control, 2006, pp. 2667-2672.

[2] M. Blanke, R. Izadi-Zamanabadi, S.A. Bogh, Z.P. Lunau, Fault-tolerant control systems - a holistic view, Control Engineering Practice 5 (5) (1997) 693-702.

[3] M. Blanke, M. Kinnaert, J. Lunze, M. Staroswiecki, Diagnosis and Fault-Tolerant Control, Springer-Verlag, 2006.

[4] G. Chesi, P. Colaneri, J.C. Geromel, R. Middleton, R. Shorten, A nonconservative LMI condition for stability of switched systems with guaranteed dwell time, IEEE Transactions on Automatic Contro 57 (5) (2012) 1297-1302.

[5] P. Colaneri, P. Bolzern, J.C. Geromel, Root mean square gain of discrete-time switched linear systems under dwell time constraints, Automatica 47 (8) (2011) 1677-1684.

[6] G. Ferrari-Trecate, M. Muselli, D. Liberati, M. Morari, A clustering technique for the identification of piecewise affine systems, Automatica 39 (2) (2003) 205-217.

[7] W. Heemels, B. De Schutter, A. Bemporad, Equivalence of hybrid dynamical models, Automatica 37 (7) (2001) 1085-1091.

[8] Y. Hirata, G. Tanaka, N. Bruchovsky, K. Aihara, Mathematically modelling and controlling prostate cancer under intermittent hormone therapy, Asian Journal of Andrology 14 (2) (2012) 270-277.

[9] R. Isermann, Fault-Diagnosis Systems, Springer Verlag, 2006.

[10] B. Jiang, M. Staroswiecki, V. Cocquempot, Fault accommodation for nonlinear dynamic systems, IEEE Transactions on Automatic Control 51 (September (9)) (2006) 1578-1583.

[11] J. Jiang, Fault-tolerant control systems — an introductory overview, Acta Automatica Sinica 31 (1) (2005) $161-174$.

[12] Z.P. Jiang, Y. Wang, Input-to-state stability for discrete-time nonlinear systems, Automatica 37 (6) (2001) $857-869$.

[13] M. Johansson, Piecewise Linear Control Systems, Springer-Verlag, 2003.

[14] M. Lazar, W. Heemels, Global input-to-state stability and stabilization of discrete-time piecewise affine systems, Nonlinear Analysis: Hybrid Systems 2 (3) (2008) 721-734.

[15] J. Löfberg, YALMIP: a toolbox for modeling and optimization in MATLAB, in: Proceedings of the IEEE Conference on Computer Aided Control Systems Design, 2004, pp. 284-289.

[16] N. Nayebpanah, L. Rodrigues, Y. Zhang, Fault detection and identification for bimodal piecewise affine systems, in: American Control Conference, 2009, pp. 2362-2366.

[17] C. Ocampo-Martinez, V. Puig, Fault-tolerant model predictive control within the hybrid systems framework: application to sewer networks, International Journal of Adaptive Control and Signal Processing 23 (8) (2009) 757-787.

[18] R.J. Patton, Fault-tolerant control systems: The 1997 situation, in: 3rd IFAC Symposium on Fault Detection Supervision and Safety for Technical Processes, vol. 3, 1997, pp. 1033-1054.

[19] Z. Ren, A. Kroll, M. Sofsky, F. Laubenstein, On identification of piecewise-affine models for systems with friction and its application to electro-mechanical throttles, in: System Identification, vol. 16, 2012, pp. 1395-1400.

[20] J.H. Richter, W. Heemels, N. van de Wouw, J. Lunze, Reconfigurable control of PWA systems with actuator and sensor faults: stability, in: 47th IEEE Conference on Decision and Control, 2008, pp. 1060-1065.

[21] J.H Richter, W. Heemels, N. van de Wouw, J. Lunze, Reconfigurable control of piecewise affine systems with actuator and sensor faults: stability and tracking, Automatica 47 (4) (2011) 678-691.

[22] T. Suzuki, N. Bruchovsky, K. Aihara, Piecewise affine systems modelling for optimizing hormone therapy of prostate cancer, Philosophical Transactions of the Royal Society A: Mathematical, Physical and Engineering Sciences 368 (1930) (2010) 5045-5059.

[23] S.M. Tabatabaeipour, M. Gholami, T. Bak, H. Schiøler, Reconfigurability of piecewise affine systems against actuator faults, in: IFAC World Congress, vol. 18, 2011, pp. 4672-4677.

[24] S.M. Tabatabaeipour, M. Gholami, K. Salahshoor, H.R. Shaker, A clustering-based bounded-error approach for identification of PWA hybrid systems, in: 9th International Conference on Control, Automation, Robotics and Vision, 2006, pp. 1-6. 
[25] S.M. Tabatabaeipour, R. Izadi-Zamanabadi, T. Bak, A.P. Ravn, Passive fault-tolerant control of discrete-time piecewise affine systems against actuator faults, Internationl Journal of Systems Scinece 43 (November (11)) (2012) 1985-1997.

[26] N. van de Wouw, A. Pavlov, Tracking and synchronisation for a class of PWA systems, Automatica 44 (11) (2008) 2909-2915.

[27] M. Vasak, N. Hure, N. Peric, Identification of a discrete-time piecewise affine model of a pitch-controlled wind turbine, in: MIPRO, 2011 Proceedings of the 34th International Convention, 2011, pp. 744-749.

[28] H. Yang, B. Jiang, V. Cocquempot, A fault tolerant control framework for periodic switched nonlinear systems, International Journal of Control 82 (1) (2009) 117-129.

[29] H. Yang, B. Jiang, V. Cocquempot, Fault tolerant control design for hybrid systems, in: Lecture Notes in Control and Information Sciences, vol. 397, Springer, 2010. 